\title{
Improving Athlete Mental Training Engagement Using Smartphone Phone Technology
}

\author{
Billymo Rist and Alan J. Pearce
}

\begin{abstract}
Elite athletes understand the benefits of mental training to deal with the stressors of training and competition. However, this particular cohort struggle with allocating time to work on mental training programs within the overall training program. The aim of this study was to test the hypothesis that Smartphone applications will improve athlete engagement with mental training programs. Forty-six male adults (mean age 24 years) who play for one professional Australian Rules football team were recruited to participate in this study. Players were randomized into three groups to undertake participation in one of three applications over a four week period: one group completed a mindfulness application (Headspace), a second group completed a brain training application (Cognifit), and a third were control and used a neutral application (Soothing Sounds). Players were assessed pre and post the four-week program on engagement; and measures including sleep (duration and quality), resilience, flow state, determination, and overall wellbeing. Results showed no differences in groups, or change in the dependent variables. However, player engagement was noticeably reduced in all groups with compliance falling, compared to initial participation levels, by $43 \%, 38 \%$ and $42 \%$ for the mindfulness, brain training and neutral applications respectively. This study demonstrates that Smartphone applications do not improve compliance with mental training programs, or significantly improve outcomes, in this professional athlete environment.
\end{abstract}

Index Terms-Elite athletes, compliance, mental training, Smartphone applications, technology.

\section{INTRODUCTION}

Achieving optimal performance in sport is a multidimensional construct whereby various factors' must work in unison in order to achieve success. In addition to physical factors, mental factors have also demonstrated to play a crucial role [1]. Elite athletes train and compete up to two to three times per day, and even as much as seven days per week [2]. Therefore, an extreme level of psychological resilience is required by elite athletes to reach the top of their respective field. The ability to consistently perform regardless of circumstance is not only required for performance during pressure moments within the match, but also to handle the daily repetitiveness of training through continually driving for improvement [3].

Manuscript received September 7, 2016; revised November 10, 2016. No financial support was provided for this study. A. J. Pearce is funded, in part, by a research grant from the Australian Football League (AFL).

B. Rist is with the St Kilda AFL club and Swinburne University of Technology Faculty of Health, Arts and Design, Melbourne, Australia (e-mail: billymo.rist@saints.com.au).

A. J Pearce is with the Swinburne University of Technology Faculty of Health, Arts and Design, Melbourne, Australia (e-mail: alanpearce@swin.edu.au)
Psychological skills, also termed mental skills, training have been identified as an important strategy to facilitate and develop an athlete's capacity to focus on performance, and to cope more effectively with negative situations [4]. Mental skills training have been identified as an important strategy to facilitate and develop an athlete's capacity to focus on performance, and to cope more effectively with negative situations [4]. Mental skills training techniques are used to develop and improve a variety of naturally occurring psychological abilities, which include attention and emotional arousal [5]. Similarly, improving essential cognitive abilities associated with peak mental functioning such as executive functioning, processing speed, and working memory have also demonstrated a significant improvement in cognitive abilities [6] that can be applied to elite athletes.

Mindfulness training has been demonstrated as an effective tool for increasing an athlete's level of mental functioning [7]. Mindfulness has its roots in Buddhist philosophy, emphasising paying attention in a particular way; purposefully, and nonjudgmentally being in the present moment [8]. Its effectiveness is thought to be because of the similarities between sensations experienced during peak performance, and mindfulness training. Both encompass a state of high concentration, an experience of a transformation of time and a certain loss of self-consciousness [8]. Further, the relaxation component of mindfulness training has been associated with enhancing an athlete's ability to mentally recover post sporting performance. This focus on the present moment has been linked to the psychology of peak performance in sport [8]. Evidence has shown that the strategy of present moment focus is associated with the likelihood of successful performance, by ensuring that unnecessary distractions which may be linked to past or future events do not inhibit momentary concentration [8]. Aherne et al [9] examined the effects of mindfulness training in a group of elite athletes over a six week period. Demonstrating that athletes who participated in mindfulness training recorded higher levels of the flow state dimensions "sense of control" and "goal clarity" during performance compared to a control group.

However, despite the growth and effectiveness of these psychological interventions, athletes and coaches fail to utilise psychological skills training due to a fear of lost time and personal expense. Ferraro \& Rush [10], for example, found that the athletes they examined $(n=20) 100 \%$ reported that they would benefit from seeing a sport psychologist, yet only $10 \%$ had engaged with one. As stated by participants they failed to access a psychologist because of a fear of lost time for physical conditioning training. In addition, participants were also concerned of the expense when consulting a psychologist [10]. Therefore, a current issue in 
sport psychology is the accessibility and efficiency of delivery methods of these psychological interventions for elite athletes.

Smartphones provide an extremely viable alternative to past methods of psychological skills training which are often described as time consuming and expensive [11]. The growth and establishment of mobile devices over the past ten years has vastly affected the user's experience. Smartphones are small, fitting into people's pockets, and carried on the person at all times, and always functioning [11]. Essential features of smartphones are applications (commonly known as 'apps'), which are downloadable software products. However, even with the dramatic increase in availability and use of smartphones and apps, little is known about their efficacy for the delivery of psychological interventions for athlete performance enhancement.

The aim of this study was to test the hypothesis that smartphone apps will increase engagement in mental training programs by athletes. A second aim of the study hypothesized that athletes who engaged with specific mental training apps will improve in areas of attention, mental well-being and mental recovery, compared to athletes who used a neutral app.

\section{METHODS}

\section{A. Participants}

Forty-six male adults $(\mathrm{M}$ age $=24$ years, $\mathrm{SD}=4.05$ years $)$ who were all elite professional athletes from one professional sporting organisation took part in the study. All were fluent in English and had no cognitive or intellectual impairment. Players were randomly assigned to one of three groups. Group 1 completed the mindfulness smartphone application $(\mathrm{n}=18$; Headspace, Version 2.0.1, Headspace Meditation Limited, UK). Group two were assigned to the cognitive brain training smartphone application $(\mathrm{n}=16$; Cognifit, Version 2.0, Cognifit, USA). Group three were assigned to a control smartphone application $(n=12$; Soothing Sounds, Version 1.2, Lost Ego Studios Limited, USA). More details regarding the smartphone apps are outlined in Section II.C.

\section{B. Procedure}

All study procedures were approved by the University Human Research Ethics committee (DUHREC 2014-105), conforming to the Declaration of Helsinki.

Following a presentation to players at one professional Australian Rules football club, participants were sent an email and invited to participate in the study. Players who consented to participation were randomly allocated a coded number for anonymity and assigned to one of three groups (see Section II.A).

Players were notified which smartphone app to download and instructed to utilise their designated smartphone application for 10 minutes per day for 28 days. No compensation for participation was offered in this study.

Prior to, and at the end of each week, players were instructed to complete an online (password protected) survey. As well as measuring frequency of engagement via completion of survey each week, the online survey consisted of five individual psychological measures: resilience, flow, sleep, determination, and wellbeing.
Resilience was measured using the Connor Davidson Resilience Scale [12]. All items pertain to the dimension of resilience, (dealing with stress, and mastery of emotions when faced with adversity). The measure consists of 10 items, (e.g. "able to adapt to change") Participants were instructed, based on how they felt after the previous week of application use to rate the strength of the agreement to each statement on a 5-point likert scale ranging from 1 (Not at all true) to 5 (True nearly all of the time). The individual scores from each question were averaged to give an overall score on resilience. The Connor Davidson Resilience Scale possesses good internal consistency with a Cronbach's alpha of .85 [12].

The ability to be "in the zone" (flow state) was measured using the Core Flow State Scale [13]. The Core Flow State Scale is a brief version, of the Long Form Flow Scale [13], made up of target items from each of the nine flow factors [13]. The Core Flow State Scale consists of 10 items (e.g., "I was switched on"). Participants were instructed, based on how they felt after the previous week of application use to rate the strength of the agreement to each statement on a 5-point likert scale ranging from 1 (Strongly Disagree) to 5 (Strongly Agree). The individual scores on each question were averaged to give an overall score on flow. The Core Flow State Scale possesses good internal consistency with a Cronbach's alpha of .92 [13].

Sleep, which was measured using the Athens Insomnia Scale [14]. The measure consists of 8 items, 5 items pertain to the nocturnal sleep dimension (e.g., "Awakening during the night") and 3 items pertain to the daytime dysfunction dimension (e.g., "Sleepiness during the day"). Participants were instructed, based on how they felt after the previous week of application use to rate the strength of the agreement to each statement on 4-point likert scale ranging from 0 (No problem) to 3 (Serious problem/did not sleep at all). The individual scores on each question were averaged to give an overall score on sleep. The Athens Insomnia Scale has good Internal consistency with a Cronbach's alpha of .89 [14].

Determination to achieve goals, which was measured using the Short Grit Scale [15]. The measure consists of 8 items, 4 items pertain to the perseverance of effort dimension (doing something despite difficulty or delay in achieving success) (e.g., "I finish whatever I begin") and 4 items pertain to the consistency of interest dimension (wanting to know or learn and achieve the desired outcome) (e.g., "I often set a goal but later choose to pursue a different one"). Participants were instructed, based on how they felt after the previous week of application use to rate the strength of the agreement to each statement on a 5 point likert scale 1 (Very much like me) to 5 (Not Like me at all). The individual scores on each question were averaged to give an overall score on determination to achieve goals. The Short Grit Scale has good internal consistency with a Cronbach's alpha of .83 [15].

Wellbeing, which was measured using the Outcome Rating Scale (ORS) [16]. The ORS is a brief alternative to the Outcome Questionnaire 45 [17], which covers a broad range of symptom and functioning domains, including subjective discomfort, interpersonal relationships, and social role performance. The ORS measure consists of 4 items (overall, individually, interpersonally, socially). Participants were instructed, based on how they felt after the previous week of 
application use to rate the strength of there agreement to each statement using a visual analogue scale of 0 (Low) to 100 (High). The individual scores on each question were averaged to give an overall score on wellbeing. The ORS has good internal consistency with a Cronbach's alpha of .90 [16].

\section{Smartphone Apps}

The mindfulness smartphone application (Headspace) consists of 10 different guided mindfulness sessions that run for approximately 10 minutes. Participants are verbally guided through the process of achieving a mindfulness state, emphasising a focus on deep breathing, relaxation of the body, and acceptance of all thoughts.

The cognitive brain training smartphone application (Cognifit) has a number of different games and puzzles, which target individual cognitive abilities such as working memory, attention and decision-making. Each challenge runs consecutively and lasts in duration of 10 minutes.

The control smartphone application (Soothing Sounds) once activated randomly plays different sounds of nature. It has no other features. Participants in this group were instructed to listen to Soothing Sounds for a 10-minute period.

\section{Data Analysis}

All data were screened for normal distribution using Shapiro-Wilk tests, and were normally distributed. All data were analysed using mixed model analysis of variance (ANOVA) with repeated measures. Where ANOVA detected differences, post-hoc comparisons were undertaken with Bonferroni adjustment. Data was analysed using SPSS V23 (SPSS Inc., USA). Descriptive data is presented as mean $( \pm$ SD). Alpha was set at $\mathrm{p}<0.05$ and effect sizes presented as partial eta squared $(\eta 2)$.

\section{RESULTS}

\section{A. Engagement with Mental Training Apps}

As shown in Fig. 1, the rate of engagement reduced in all three groups. There was a $43 \%$ dropout in the Cognifit group; $38 \%$ dropout in the Headspace group, and a $42 \%$ dropout in the control group.

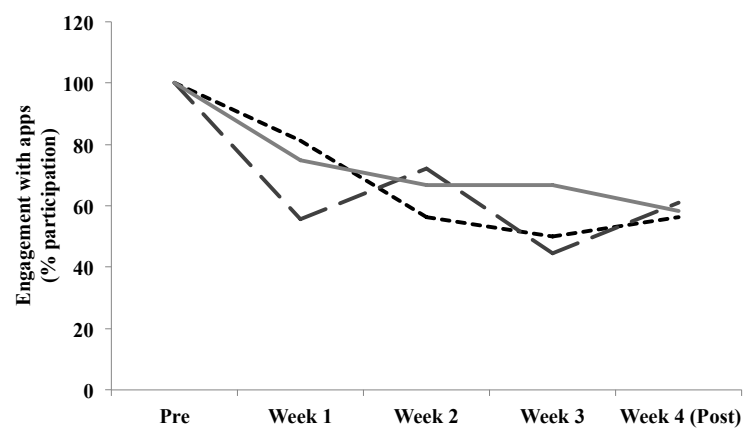

Fig. 1. Engagement in apps over duration of study.

Further, compliance with mental training evaluations (Table I) showed no differences between the three groups $(F(2,43)=0.308, p=0.737)$.

\section{B. Individual Psychological Measures}

Resilience (Table II) showed no significant interaction between the different smartphone applications, and time $(F(6,38)=0.787, p=0.823)$. There was no significant main effect for time $(F(3,19)=0.302 p=0.586, \eta 2=0.046)$ or group $(F(2,21)=0.723, p=0.23, \eta 2=0.30)$.

TABLE II: MEAN ( \pm SD) NUMBER OF EVALUATIONS COMPLETED (MAX 4)

\begin{tabular}{cccc}
\hline & Cognifit & Headspace & Control \\
\hline \hline $\begin{array}{c}\text { Completed } \\
\text { evaluations }\end{array}$ & $2.4( \pm 1.4)$ & $2.7( \pm 1.0)$ & $2.6( \pm 1.2)$ \\
\hline
\end{tabular}

TABLE 2: MEAN ( \pm SD) FOR RESILIANCE MEASURES (MAX 5)

\begin{tabular}{lccc}
\hline \hline & Cognitfit & Headspace & Control \\
\hline End of week 1 & $4.1( \pm 0.4)$ & $3.6( \pm 0.4)$ & $3.9( \pm 0.5)$ \\
End of week 2 & $3.9( \pm 0.4)$ & $3.8( \pm 0.3)$ & $4.0( \pm 0.4)$ \\
End of week 3 & $3.9( \pm 0.4)$ & $3.6( \pm 0.4)$ & $4.0( \pm 0.4)$ \\
End of week 4 & $3.8( \pm 0.3)$ & $3.7( \pm 0.3)$ & $3.9( \pm 0.5)$ \\
\hline \hline
\end{tabular}

There was no significant interaction for flow between the different smartphone applications, and time $(F(6,38)=.793$, $p=.334)$. There was no significant main effect for time $(F(3$, $19)=1.207 p=.581, \eta 2=.160)$ or group $(F(2,21)=0.648$, $p=.118 \eta 2=0.184)$.

TABLE III: MEAN ( \pm SD) FOR FLOW MEASURES (MAX 5)

\begin{tabular}{lccc}
\hline \hline & Cognitfit & Headspace & Control \\
\hline End of week 1 & $3.8( \pm 0.5)$ & $3.8( \pm 0.5)$ & $3.5( \pm 0.4)$ \\
End of week 2 & $3.6( \pm 0.4)$ & $3.7( \pm 0.6)$ & $3.1( \pm 0.5)$ \\
End of week 3 & $3.8( \pm 0.4)$ & $3.5( \pm 0.5)$ & $3.6( \pm 0.4)$ \\
End of week 4 & $3.7( \pm 0.6)$ & $3.6( \pm 0.5)$ & $3.6( \pm 0.6)$ \\
\hline \hline
\end{tabular}

Overall sleep data incorporating both quality and duration, where lower numbers mean better sleep is illustrated in Table IV). Results showed no significant interaction between the different smartphone applications and time $(F(6,38)=1.721$, $p=0.049)$. There was no significant main effect for time $(F(3$, $19)=3.148 p=0.143$, partial eta squared $=0.332$ ) or group $(F(2,21)=0.286, p=0.239, \eta 2=0.127)$.

Determination (Table V) showed no significant interaction between the different smartphone applications, and time $(\mathrm{F}$ $(6,38)=0.601, p=0.668$.). There was no significant main effect for time $(F(3,19)=0.528 p=.728, \eta 2=0.077)$ or group $(F(2,21)=0.272 p=.250, \eta 2=0.124)$.

TABLE IV: MEAN ( \pm SD) FOR SLEEP MEASURES (MAX 3)

\begin{tabular}{lccc}
\hline \hline & Cognitfit & Headspace & Control \\
\hline End of week 1 & $0.6( \pm 0.4)$ & $0.6( \pm 0.5)$ & $0.7( \pm 0.4)$ \\
End of week 2 & $0.7( \pm 0.4)$ & $0.4( \pm 0.2)$ & $0.6( \pm 0.4)$ \\
End of week 3 & $0.6( \pm 0.4)$ & $0.7( \pm 0.5)$ & $1.1( \pm 0.4)$ \\
End of week 4 & $0.6( \pm 0.6)$ & $0.5( \pm 0.7)$ & $0.7( \pm 0.6)$ \\
\hline \hline
\end{tabular}

TABLE V: MEAN ( \pm SD) FOR DETERMINATION MEASURES (MAX 5 )

\begin{tabular}{lccc}
\hline \hline & Cognitfit & Headspace & Control \\
\hline End of week 1 & $3.8( \pm 0.5)$ & $3.7( \pm 0.3)$ & $3.7( \pm 0.4)$ \\
End of week 2 & $3.6( \pm 0.6)$ & $3.7( \pm 0.3)$ & $3.5( \pm 0.4)$ \\
End of week 3 & $3.5( \pm 0.4)$ & $3.9( \pm 0.5)$ & $3.5( \pm 0.5)$ \\
End of week 4 & $3.8( \pm 0.4)$ & $3.8( \pm 0.4)$ & $3.7( \pm 0.6)$ \\
\hline \hline
\end{tabular}

Wellbeing (Table VI) showed no significant interaction between the different smartphone applications and time $(F(6,38)=1.294, p=0.671)$. There was no significant main effect for time $(F(3,19)=0.525 p=0.283, \eta 2=0.077)$, or 
group $(F(2,21)=0.245 p=0.243, \eta 2=0.126)$.

TABLE VI: MEAN ( \pm SD) FOR WELLBEING MEASURES (MAX 100)

\begin{tabular}{llll}
\hline \hline & Cognitfit & Headspace & Control \\
\hline End of week 1 & $79.8( \pm 11.7)$ & $76.9( \pm 6.5)$ & $82.7( \pm 9.6)$ \\
End of week 2 & $72.6( \pm 9.4)$ & $79.7( \pm 7.5)$ & $85.6( \pm 8.2)$ \\
End of week 3 & $79.6( \pm 6.1)$ & $73.3( \pm 11.1)$ & $78.8( \pm 11.8)$ \\
End of week 4 & $74.8( \pm 13)$ & $75.2( \pm 13.9)$ & $78.7( \pm 10.9)$ \\
\hline \hline
\end{tabular}

\section{Discussion}

The present study examined whether the use of an alternative medium of delivery (smartphone apps) would improve engagement in mental skills training in an elite athlete population. The second objective was to examine the effectiveness of mindfulness and cognitive brain training apps on various individual psychological measures that influence training and competitive performance.

Both mindfulness and cognitive brain training have previously been demonstrated to be effective interventions in enhancing mental functioning [5], [6]. Combined with efficient and easy to access smartphone technology, it was hypothesized that athletes would engage with these apps (compared to control) and as a result, show effects across a range of psychological attributes.

However, results indicated that there was no difference in engagement between any of the smartphone apps as well as completion of the online surveys across the duration of the study. Furthermore, there was no significant difference between the mindfulness or cognitive brain training groups compared to each other and to a control application on any of the measures (resilience, flow, sleep, determination to achieve goals or wellbeing) over the four time points. Thus, neither of the hypotheses was supported.

To date no previous research has examined psychological skills training for athletes using non-conventional methods of delivery, such as smartphone apps. Given that athletes, and coaching staff, regularly complain that psychological skills and mental training are difficult to fit in due to time and costs [10], the use of trialing smartphone applications would abate these barriers. Smartphone technology is growing at a rapid rate and is efficient and accessible [11]. The results of the current study are contrary to that of previous research that has investigated the effect of mental skills training [5]. It should be noted, however, that the present study differed from [5]. The present study was only able to undertake a four-week mental skills program, rather 16 week [5]. However, the rapid decrease in engagement suggests that a longer intervention may not have yielded any further differences between this previous [5] and present study.

Nouchi et al. [6] using an intervention of four-weeks duration, demonstrated a significant improvement in cognitive functions such as working memory, and processing speed amongst young non-athlete adults from the general population over a four-week period. This study was not able to replicate these findings. It may be that the population utilized (non-athlete versus professional athletes) may have contributed to the disparate findings. It should be also noted that these authors [6] ensured compliance by measuring participants before and after every intervention. Our study did not undertake daily measures, as the primary aim of the study was to investigate if professional athletes would be responsible for their own mental training, and that smartphone apps would engage athletes to regularly undertake the training.

\section{CONCLUSION}

In conclusion, this study demonstrated that smartphone apps did not retain engagement in a professional athlete population. The loss in retention by the athletes demonstrated what circumstances athletes will best interact and utilise psychological skills training to improve wellbeing and performance. Elite athletes require significant structure [2], [3], to complete their required daily tasks that encompass being a professional athlete. It may be that smartphone apps do indeed have an effect, however, coaching staff will need to schedule specific time into their training programs to ensure mental skills training is habit forming in their athletes, just like athletes make it a habit to complete physical training programs for strength, stamina, flexibility, and recovery [18].

Further studies examining smartphone application technology, and in particular how best to retain engagement, will assist sports psychologists and coaches into how elite athletes mindsets can be further improved to achieve the mental edge, which is required to achieve ultimate success in the elite sporting environment.

\section{ACKNOWLEDGMENT}

The authors would like to acknowledge St Kilda AFL club for their participation in this study.

\section{REFERENCES}

[1] L. Behncke. Mental skills training for sports: A brief review. Journal of Sport Psychology. [Online]. Available: https://athleticinsight.com/Vol6Iss1/SkillsPDF.pdf

[2] R. Budgett, "Fatigue and underperformance in athletes: Overtraining syndrome," British Journal of Sports Medicine, vol. 32, pp. 107-110, June 1998.

[3] R. E. Vetter and M. L. Symonds, "Correlations between injury, training intensity, and physical and mental exhaustion among college athletes," Journal of Strength and Conditioning Research, vol. 24, pp. 587 - 596, March 2010.

[4] F. L. Gardner, Z. E Moore, and Z. E, "A Mindfulness-acceptance-commitment-based approach to athletic performance enhancement: theoretical considerations," Behaviour Therapy, vol. 35, pp. 707-723. November 2004.

[5] D. J. Edwards and S. D. Edwards, "The evaluation of a psychological skills training programme for Rugby players," African Journal for Physical, Health Education, Recreation and Dance, vol. 18, pp 525-534. September 2012.

[6] R. Nouchi, Y. Taki, H. Takeuchi, H. Hashizume, T. Nozawa, T. Kambara, A. Sekiguchi, C. M. Miyauchi, Y. Kotozaki, H. Nouchi, and R. Kawashima, "Brain training game boosts executive functions, working memory and processing speed in the young adults: A randomised control trial," PloS one, vol. 8, February 2013.

[7] J. Carmody and R. A. Baer, "Relationships between mindfulness practice and levels of mindfulness, medical and psychological symptoms and well-being in a mindfulness-based stress reduction program," Journal of Behavioral Medicine, vol. 31, pp. 23-33, February 2008.

[8] H. Y. Kee, and C. K. J. Wang, "Relationships between mindfulness flow dispositions and mental skills adoption: A cluster analytic approach," Psychology of Sport and Exercise, vol. 9, pp. 393-411, July 2008.

[9] C. Aherne, A. P. Moran, and C. Lonsdale, "The effect of mindfulness training on athletes' Flow: An initial investigation," The Sports Psychologist, vol. 25, pp. 177-189, June 2011.

[10] T. Ferraro and S. Rush, "Why athletes resist sport psychology," Athletic Insight, vol. 2, pp. 9-14, October 2000. 
[11] C. Kratzke and C. C. Cox, "Smartphone technology and apps: Rapidly changing health promotion," International Electronic Journal of Health Education, vol. 15, pp. 72-82, January 2012.

[12] S. Vaishnavi, K. Connor, and J. R. Davidson, “An abbreviated version of the connor-davidson resilience scale (CD-RISC), the CD-RISC2: Psychometric properties and applications in psychopharmacological trials," Psychiatry Research, vol. 152, pp. 293-297, August 2007.

[13] S. A. Jackson, R. C. Eklund, and A. J. Martin, The Flow Manual: The Manual for the Flow Sclaes, Queensland, Australia: Mind Garden Inc, 2010.

[14] C. R. Soldatos, D. G. Dikeos, and T. J. Paparrigopoulos, "Athens Insomnia Scale: Validation of an instrument based on ICD-10 criteria," Journal of Psychosomatic Research, vol. 45, pp. 555-560, June1999.

[15] A. L. Duckworth and P. D. Quinn, "Development and validation of the Short Grit Scale (GRIT-S)," Journal of Personality Assessment, vol. 91, pp. 166-174. February 2009.

[16] S. D. Miller, B. L. Duncan, J. Brown, J. A Sparks, and D. A. Claud, "The outcome rating scale: A preliminary study of the reliability, validity, and feasibility of a brief visual analog measure," Journal of Brief Therapy, vol. 2, pp. 91-100. January 2003.
[17] R. Mueller, M. J. Lambert, and G. M. Burlingame, "Construct validity of the Outcome Questionnaire: A confirmatory factor analysis," Journal of Personality Assessment, vol. 2, pp. 248-262, April 1998.

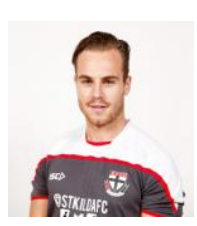

Billymo Rist is the player development coordinator at the Kilda Football Club in the Australian Football League .

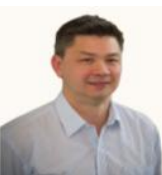

Alan Pearce is an adjunct associate professor in the Faculty of Health, Arts and Design, Swinburne University; and adjunct senior research fellow at Melbourne School of Health Sciences, The University of Melbourne. 